\title{
Wear Behavior of Ti6AI4V Surfaces Functionalized through Ultrasonic Vibration Turning
}

\author{
R. Bertolini (iD, A. Ghiotti, and S. Bruschi
}

Submitted: 7 February 2021 / Revised: 21 May 2021 / Accepted: 29 May 2021 / Published online: 18 June 2021

\begin{abstract}
Even if titanium alloys are widely used for biomedical applications, the tendency they show to wear is a matter of concern for their durability. In this research work, Ultrasonic Vibration Turning (UVT) was used to generate texturized surfaces on Ti6Al4V cylinders for improving their wear performances. To prove that, in vitro wear tests were performed, during which the UVT-ed Ti6Al4V cylinders were made to slide against polyethylene disks to replicate a currently used biomedical pair. It was shown that the UVT-ed Ti6AI4V cylinders were characterized by enhanced performances in terms of coefficient of friction and wear rate, which were reduced to 52 and $25 \%$, respectively, compared to polished Ti6Al4V surfaces.
\end{abstract}

Keywords abrasive wear, friction coefficient, surface texture, surface integrity, Ti6A14V, ultrasonic vibration turning

\section{Introduction}

Ti6Al4V titanium alloy is widely used for biomedical applications because of its excellent combination of high strength, low density, and corrosion resistance. Unfortunately, Ti6Al4V alloy is known to possess poor wear resistance that may restrict its applications particularly in those involving high wear and friction. As example, Molinari et al. (Ref 1) studied the Ti6Al4V dry sliding wear behavior at varying velocity and load: the Ti6Al4V low wear resistance was attributed to the poor protection exerted by the surface oxide, which was brittle and tended to be continuously fragmented (Ref 2 ). This points out the need to develop robust strategies to improve the wear resistance of the titanium alloys. Up to now, several approaches have been applied for this aim, among which it is worth citing thermal oxidation (Ref 3 ) and treatments (Ref 4$)$, coatings (Ref 5), mechanically based methods (Ref 6), and more lately grafting (Ref 7). The latter technique deals with the modification of bearing surfaces with a hydrophilic polymer layer possessing a brush-like structure, assumed to be similar to that of the articular cartilages.

Another promising technique is micro-texturing, which has been already used to reduce friction and wear of biomedical articulating surfaces. Ito et al. (Ref 8) manufactured circular dimples of $0.5 \mathrm{~mm}$ diameter and $0.1 \mathrm{~mm}$ depth, with a $1.2 \mathrm{~mm}$ pitch on a $\mathrm{CoCr}$ femoral head, observing a $17 \%$ friction reduction and a $36 \%$ reduction in the polyethylene wear, and hypothesizing that this might be the result of the abrasive wear particles being trapped in the dimples, and the lubricant being accumulated and subsequently provided from the dimples to the contact surface during lubricant starvation conditions.

R. Bertolini, A. Ghiotti, and S. Bruschi, Department of Industrial Engineering, University of Padova, via Venezia 1, Padova, Italy. Contact e-mail: rachele.bertolini@unipd.it.
Sawano et al. (Ref 9) used a water jet cutting to machine 0.25-4.4- $\mu \mathrm{m}$-deep channels, spaced $10 \mu \mathrm{m}$ apart and running perpendicular to the direction of articulation, on a CoCrMo plate: an effective reduction in the polyethylene wear was measured during pin-on-disk experiments in case of plates with 0.9 - $\mu$ m-deep dimples, which was attributed to the wear particles entrapment inside the channels. Laser surface texturing was used by Chyr et al. (Ref 10) to generate different micro-textures on the surface of a CoCrMo plate, demonstrating that, thanks to the increase in the lubricant thickness, the coefficient of friction was reduced. Hu et al. (Ref 11) applied laser surface texturing to form regular dimples of different size and different spacing on Ti6Al4V surfaces. Pin-on-disk tests were carried out against steel using two lubricants with different kinetic viscosity, observing that the textured surfaces exhibited lower friction coefficient and wear compared with the un-textured surface, thanks to the hydrodynamic pressure increase as a consequence of the higher film thickness in correspondence of the dimples. Diode pumped solid-state laser was exploited for producing micro-groove geometries and promotes cell adhesions in the work of Fasasi et al. (Ref 12). In (Ref 13), coating was coupled to texturing to improve wear resistance of stainless steel hip prosthesis heads. Two types of coating, namely hydrogenated amorphous carbon (a-C:H) and tetrahedral amorphous carbon (Ta-C), were magnetron sputtered on the dimples surfaces. Tribology tests carried out against ceramic ball showed a significant reduction in the friction coefficient for the coated surfaces compared to the base stainless steel surface, with a-C:H samples showing the best performances.

Besides improving the tribological performances, surface texturing can be a potential strategy to deliver anti-inflammatory drugs through their storage in the textured micro-dimples and subsequent release in situ (Ref 14). This can be helpful in facing infections that may lead to premature postoperative revisions of the implant (Ref 15, 16).

Ultrasonic Vibration Turning (UVT) represents a way for texturing surfaces by means of ultrasonic vibration of the tool during machining that allows the creation of dimples on the machined surface. Greco et al. (Ref 17) showed that the dimples size was based on the tunable oscillation of the tool path, besides being applicable to different surfaces, like outer 
and inner cylindrical surfaces, flat/end faces. Liu et al. (Ref 18) investigated the effect of the UVT process parameters, including clearance angle, amplitude, and cutting speed, on the dimples cross-sectional profile along the cutting direction: they proposed a method to predict the dimples arrangement and overall textured surface topography. Besides uni-directional, also bi-dimensional elliptical vibration was applied to fabricate more complex micro-textures on cylindrical surfaces, as was in (Ref 19).

Laser texturing represents the most adopted process for texturing; it uses localized melt and vaporization of the material, to form individual rounded dimple depressions (Ref 20). This method, besides needing a laser and optical systems that are costly and require clean environments, induces on the worked surface a thermally affected zone usually associated with white layer formation, which impairs the mechanical characteristics of the surface itself. Actually, the material near the dimples is subjected to very high temperatures causing microstructural changes, residual thermal stresses, and bulges formation that makes mandatory post-processing techniques to remove the white layer.

On the contrary, UVT is capable of generating highly precise textures even on complex surfaces with minimal induced surface damage. In addition, texturing can be obtained simultaneously to finishing machining operations that are usually required for metal components of hip implants (Ref 21). For these reasons, it appears as the ideal process to minimize time and cost, thus being sustainable from manufacturing companies.

A few researchers have attempted to apply UVT to enhance the tribological performances of functional surfaces, but none of them have targeted biomedical applications. Amini et al. (Ref 22] studied the effect of the machining parameters and vibration mode on the tribological properties of the AA7075/ chrome steel pair, showing that the textured surfaces were characterized by both lower coefficient of friction and wear rate compared to not-textured surfaces. Xing et al. (Ref 23) examined the tribological properties of aluminum alloy surfaces resulted from ultrasonic vibration-assisted milling compared to conventional surfaces, showing a $20 \%$ decrease in friction and a $140 \%$ increase in oil retention.

UVT is here presented for the first time as a method to realize textures that can be exploited to prevent excessive wear, and, therefore, increase durability of the femoral head of hip prostheses. Dimples were generated on the surface of Ti6Al4V cylinders that, afterwards, were tested in a cylinder-on-disk configuration against Ultra-High-Molecular-Weight-Polyethylene (UHMWPE) as counterpart material. Results were then compared with the ones obtained by using Ti6Al4V smooth surfaces, which represent the baseline for comparison.

\section{Experimental}

\subsection{Materials}

In this study, the ISO5834/2 resin GUR ${ }^{\mathrm{TM}} 1050$ UHMWPE was provided in form of bars of $60 \mathrm{~mm}$ width and $1000 \mathrm{~mm}$ length. The UHMWPE bars were machined in order to get a surface roughness comparable to that of commercially available acetabular cups (average surface roughness $\mathrm{Sa}$ equal to $0.97 \pm 0.04 \mu \mathrm{m}$ as shown in Fig. 1(a)). After a roughing step to achieve $40 \mathrm{~mm}$ of diameter, a face turning finishing operation was carried out, using feed of $0.05 \mathrm{~mm} / \mathrm{rev}$ and cutting speed of $80 \mathrm{~m} / \mathrm{min}$. Finally, disks of $5 \mathrm{~mm}$ thickness were cut from the machined bars, as shown in Fig. 1(b).

The Ti6Al4V titanium alloy was provided in form of bars of $40 \mathrm{~mm}$ width and $180 \mathrm{~mm}$ length, from which cylinders were machined to be used later on in the wear experiments.

Table 1 reports the main mechanical characteristics of the materials used in the study.

\subsection{Machining Tests}

The machining tests were carried out on a Mori Seiki ${ }^{\mathrm{TM}} \mathrm{NL}$ $1500 \mathrm{CNC}$ lathe equipped with an UVT apparatus. The UVT setup (see Fig. 2a) basically consists of a piezoelectric transducer, a sonotrode, a tool tip, a mechanical setup to fit in the turret of the lathe, and driving electronics. As can be seen from the scheme of Fig. 2(b), the cutting tool vibrates perpendicularly to the feed direction. More details about the experimental apparatus can be found in (Ref 24). For all the machining tests of this research, the vibration frequency was kept fixed and equal to $30 \mathrm{kHz}$.

A DCGT 11 T3 08-UM GC1105-coated tungsten carbide cutting tool was used. A fresh cutting edge was adopted for each test, in order to avoid the influence of the tool wear on the machined surface characteristics.

Before UVT, the Ti6Al4V bars were subjected to a turning pass to achieve a diameter of $19.3 \mathrm{~mm}$, using the same UVT system where the ultrasonic vibration was turned off. Later on, the ultrasonic vibration was turned on to carry out a finishing pass, where a depth of cut equal to $0.05 \mathrm{~mm}$ was set to reach a final diameter of $19.2 \mathrm{~mm}$. In order to evaluate the effect of the dimples shape on the Ti6Al4V wear behavior, different cutting speeds were adopted, namely $100 \mathrm{~m} / \mathrm{min}$ and $200 \mathrm{~m} / \mathrm{min}$, whereas the feed was kept fixed and equal to $0.05 \mathrm{~mm} / \mathrm{rev}$. The tests were performed under dry condition and repeated six times for each cutting parameter.

Since biomedical surfaces are usually polished before being put in-service, a few Ti6Al4V cylinders were ground after conventional turning to achieve a mirror-like surface roughness. For doing that, the cylinders were ground using up to $4000 \mathrm{SiC}$ grit paper and then polished by using a $3-\mu \mathrm{m}$ diamond paper and $\mathrm{SiO}_{2}$ colloidal dispersion in demineralized water and $\mathrm{H}_{2} \mathrm{O}_{2}$, achieving a surface roughness $(\mathrm{Sa})$ of $0.057 \pm 0.007 \mu \mathrm{m}$.

Table 2 summarizes the experimental plan adopted for the machining tests.

\subsection{Characterization After Machining}

Vickers micro-hardness was measured using a microhardness tester with a load of $10( \pm 0.5)$ gr for $30 \mathrm{~s}$; three values were recorded for each measurement point and the average value reported. The hardness measurements were taken every $20 \mu \mathrm{m}$ from the machined surface to a depth of $200 \mu \mathrm{m}$.

The surface of a biomaterial is the first contact between the implant and the biological cells or fluids, and its characteristics, among which the surface wettability plays the major role, influence the biocompatibility degree (Ref 25). The measure of the contact angle to evaluate the wettability of the Ti6Al4V machined cylinders was carried out applying a static sessile drop technique. The experimental apparatus consists of a horizontal stage used to place the sample, allowing the specimen adjustment in the z-direction, a micrometer syringe 

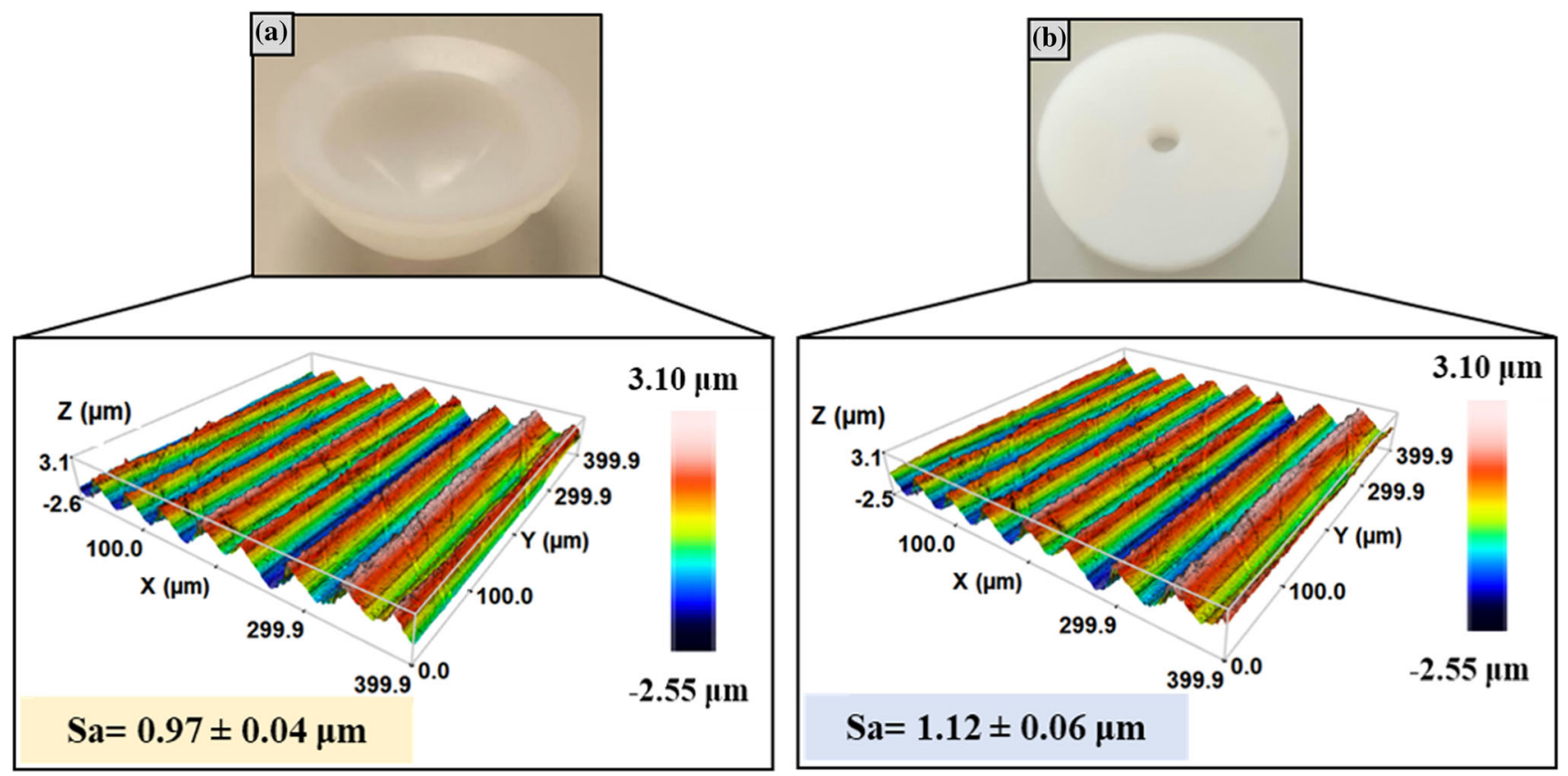

Fig. 1. Surface topography of a commercially available acetabular cup (a) and of a machined UHMWPE disk (b)

to deliver a liquid drop, a halogen and intensity adjustable light source to illuminate the sample, and a camera for the images acquisition. Before measuring, the samples were cleaned in an ultrasonic bath for $15 \mathrm{~min}$ to remove any residue. A liquid droplet of distilled water of $10 \mu \mathrm{L}$ volume was let fall on the lateral surface of the cylindrical sample. The measurements were conducted at room temperature $\left(25^{\circ} \mathrm{C}\right)$; each experiment was repeated eight times to assure reproducibility and the mean value of the experimental data calculated.

Since the surface topography has a decisive influence on the tribological performances, it needs to be carefully investigated (Ref 26). The surface topography of the Ti6Al4V machined cylinders was inspected using a Plu Neox ${ }^{\mathrm{TM}}$ optical profiler with a $20 x$ magnification confocal objective. Data processing, filtering, and evaluation of the surface texture parameters were performed according to the ISO 25178 series (Ref 27). On the basis of a previous experimental work (Ref 28), the following surface texture parameters were considered:

- Arithmetical mean height of the scale-limited surface (Sa), the most popular areal parameter, which represents the baseline for comparison with other research works.

- Reduced dale height (Svk), an effective parameter to describe the relevance of valleys that can affect the fluid retention in wet applications.

- Reduced peak height (Spk), a parameter giving information about the damaging top portions of the surface that influence the initial sliding during wear tests and where the peaks are gradually worn out.

- Skewness of the scale-limited surface (Ssk), showing whether a surface is dominated by peaks or valleys, thus measuring the symmetry of the profile about the mean line.

Besides the aforementioned surface texture parameters, two additional indicators were taken into account, namely the Aspect Ratio (AR) of the dimples and the density of the dimples (Sdd), the latter introduced here for the first time with the aim of characterizing the UVT-induced texture.

$A R$ refers to the ratio between the dimples' depth and mean diameter. The dimples average depth was calculated on the basis of three measurements of depth taken on the 2D profile, which was extracted from the 3D topography of the machined surface at six fixed coordinates. Three different topographies were considered for such calculation for each cutting condition. The dimples diameter $d_{\text {gap }}(\mu \mathrm{m})$ is considered equal to the distance between two subsequent dimples and can be expressed through Eq 1:

$d_{\text {gap }}=\frac{V_{c} * 10^{6}}{60 f}$

where $V c(\mathrm{~m} / \mathrm{min})$ is the cutting speed and $f(\mathrm{~Hz})$ the ultrasonic frequency.

The density of the dimples (Sdd) refers to the number of dimples per unit area, and is equivalent to the density of the peaks parameter (Spd), but evaluated on a topography symmetrical with respect to the $\mathrm{x}$-axis.

\subsection{Wear Tests}

To investigate the tribological performances of the couple Ti6Al4V-UHMWPE, the Universal Rtec ${ }^{\mathrm{TM}}$ tribometer was used. A cylinder-on-disk configuration, in which a Ti6Al4V cylinder was made to slide on a UHMWPE disk, was adopted for the wear tests. A circular multidirectional motion was preferred to linear reciprocating motion in order to mimic the quasi-elliptical path encountered during the in vivo gait (Ref 29) and to prevent the alignment of the polymer chains and its consequent strain hardening (Ref 30 ). In order to reproduce the human body environment, the wear tests were performed in saline solution at $37 \pm 1{ }^{\circ} \mathrm{C}$, constantly monitored through a thermometer immersed in a basin filled with water. Saline solution was considered as liquid medium because it creates tougher wear conditions compared to the serum solution, which 
Table 1 Mechanical characteristics of the tested materials in the as-delivered condition according to the suppliers' datasheets

\begin{tabular}{|c|c|c|c|c|c|}
\hline & $\mathbf{E}, \mathbf{G P a}$ & UTS, MPa & $\mathbf{Y}, \mathbf{M P a}$ & Elongation, \% & Poisson's ratio, - \\
\hline \multirow[t]{2}{*}{ Ti6A14V } & 120 & 1020 & 950 & 14 & 0.35 \\
\hline & E, GPa & Density, $\mathrm{g} / \mathrm{cm}^{3}$ & $\mathbf{Y}, \mathbf{M P a}$ & Izod notched, $\mathrm{kJ} / \mathrm{m}^{2}$ & Poisson's ratio, - \\
\hline GUR 1050 UHMWPE & 1.2 & 0.93 & 0.48 & $>73$ & 0.46 \\
\hline
\end{tabular}

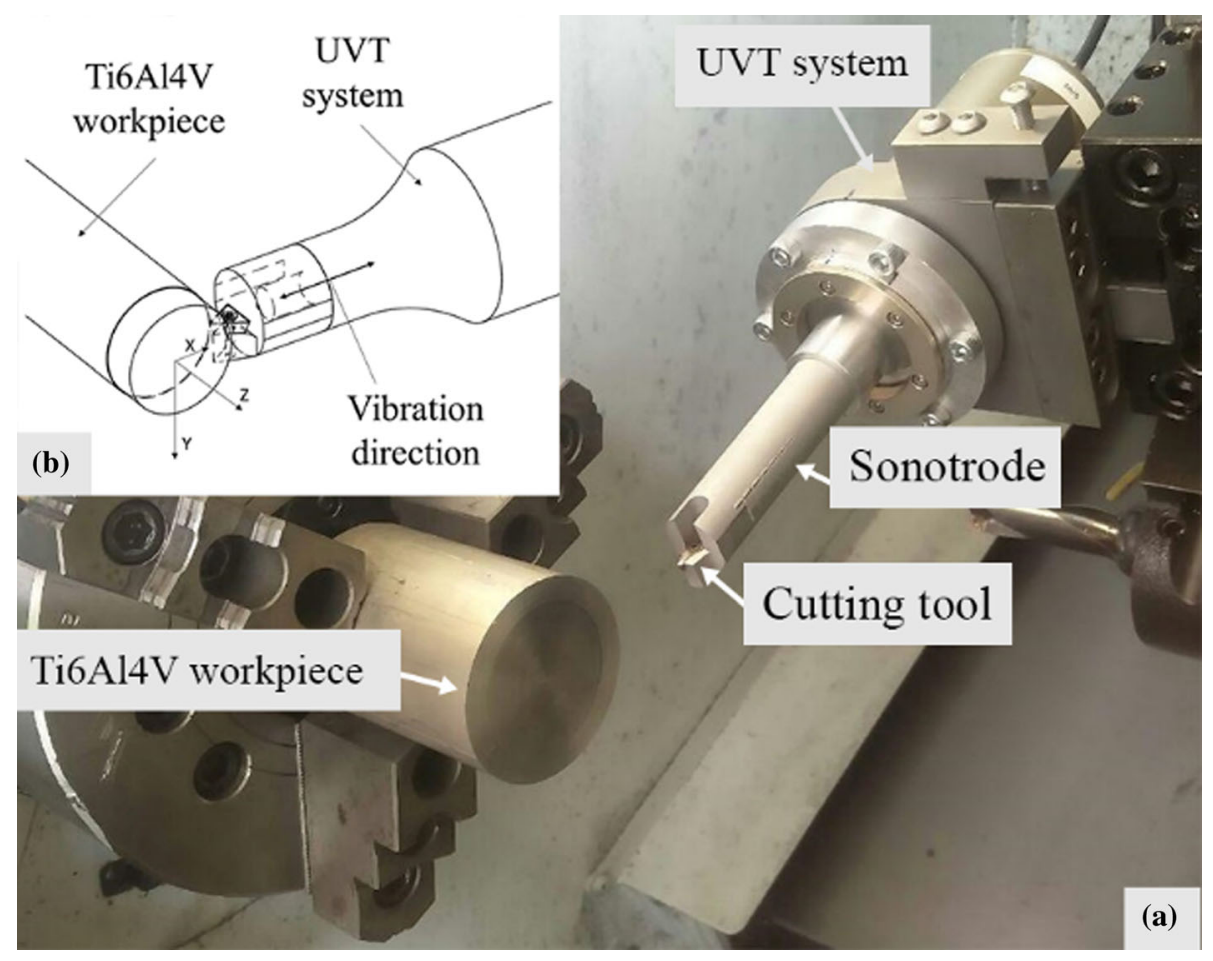

Fig. 2. Photo (a) and scheme (b) of the UVT set-up

is a better lubricant (Ref 31). The experimental setup for the wear tests is shown in Fig. 3(a).

During testing, the load was changed to better simulate the load variation during the human gait. In particular, the circular motion, with a radius of $14 \mathrm{~mm}$ at angular speed of $50.3 \mathrm{rad} / \mathrm{s}$, was divided into two parts in which the load was set to $20 \pm 1$ and $40 \pm 1 \mathrm{~N}$, respectively, corresponding to a maximum Hertzian contact pressure of 15.8 and $22.3 \mathrm{MPa}$ accordingly to the Hertz's theory (Ref 32). Since the wear configuration was cylinder-on-disk, the contact pressure was applied on a line. The chosen force values were in agreement with the calculations made by Borruto (Ref 33 ), assuming a safety factor of 3 and 6 , respectively, taking into account possible strikes to the joint.

With the aim to evaluate the resistance to wear of the textured Ti6Al4V samples, two different typologies of wear tests were carried out, here called "one-month test" and "threemonth test." The first lasted 94 minutes, equivalent to a period of one month after implantation, while the second type of test lasted 282 minutes, equal to three months after implantation. To reduce the test duration, the load frequency was speed up to 8 $\mathrm{Hz}$; in any case, this fixed value of load frequency does not interfere with the experiment outcomes according to the ISO $7206 / 3$ standard (Ref 34).
The one-month test was performed to evaluate the resistance to wear of the textured samples, since this texture can be exploited to store drugs to fight early postoperative infections, which may develop during the first month after implantation (Ref 35).

On the other hand, the three-month tests were performed to investigate the actual wear performances of the textured samples, since wear after one-month test was almost negligible and, therefore, impossible to be measured precisely.

Furthermore, to consider the oscillatory characteristic typical of human movements, a variation in the rotational direction was adopted, as half cycle under a given load was made counter clockwise. A scheme of the adopted wear tests is reported in Fig. 3(b).

The wear tests were repeated three times for each condition.

\subsection{Characterization After Wear Testing}

The surface topography of the worn surfaces of both the Ti6Al4V cylinders and UHMWPE disks was evaluated using the $3 \mathrm{D}$ optical profiler. For the latter, the topographies were taken in correspondence of four referenced points. Then, in order to assess the wear volume, 2D profiles of the wear track were extrapolated, and the area of the wear track was 
calculated. The average cross section of the worn area was then multiplied by the circumference length in order to assess the wear volume of the UHMWPE.

The FEI QUANTA 450 ${ }^{\mathrm{TM}}$ Scanning Electron Microscope (SEM), equipped with the Everhart-Thornley Detector (ETD) and the Back Scattered Electron (BSE) one, was used to examine the surface characteristics of both the worn Ti6Al4V cylinders and UHMWPE disks. Before inspection at SEM, the UHMWPE disks were coated with gold to make them conductive. The gold coating was deposited using a vacuum machine, with a current of $15 \mathrm{~mA}$ for $120 \mathrm{~s}$. The material adhesion within dimples was evaluated by means of an $\mathrm{EDAX}^{\mathrm{TM}}$ dispersive $\mathrm{X}$-ray spectroscopy detector (EDS), through a point analysis conducted at an acceleration voltage of $20 \mathrm{kV}$.

\section{Results and Discussion}

\subsection{Characteristics of the Machined Samples Before and After Wear Testing}

The micro-hardness data shown in Fig. 4 prove that no evident hardening characterizes any of the machined samples. All the measured hardness values are, in fact, comparable to that of the bulk region. The absence of strain hardening was expected in the polished sample as the heat arisen in the cutting zone softens the effect of strain hardening induced by cutting. Similarly, the absence of strain hardening in the UVT-ed samples can be attributed to the fact that the temperature

Table 2 Experimental plan adopted for the machining tests

\begin{tabular}{lcc}
\hline Test ID & Finishing operation & Cutting speed, m/min \\
\hline Smooth & Polishing & $\backslash$ \\
UVT-100 & UVT & 100 \\
UVT-200 & UVT & 200 \\
\hline
\end{tabular}

increase due to the tool ultrasonic motion exceeded the strain hardening effect due to cutting. Similarly,

Figure 5 shows water droplets made to fall on the cylindrical surfaces as a function of the machining parameters. Surface wettability is an important issue since it play a fundamental role in the biological response of the implant/bone and implant/soft tissue interfaces (Ref 36). As the obtained contact angles are strictly related to the surface topography, it was investigated if the machining-induced topography could affect in a negative way the hydrophilic nature of titanium. The obtained results show that the contact angle was not affected by different surface topographies; therefore, it can be stated that UVT did not alter the Ti6Al4V wettability characteristics.

As shown in Fig. 5, only slight differences were obtained among the UVT-ed samples. In fact, the cutting speed determines the dimple gaps in the cutting direction, thus having a limited impact on the contact angle in the feed direction. These findings are confirmed by a previous research study (Ref 37).

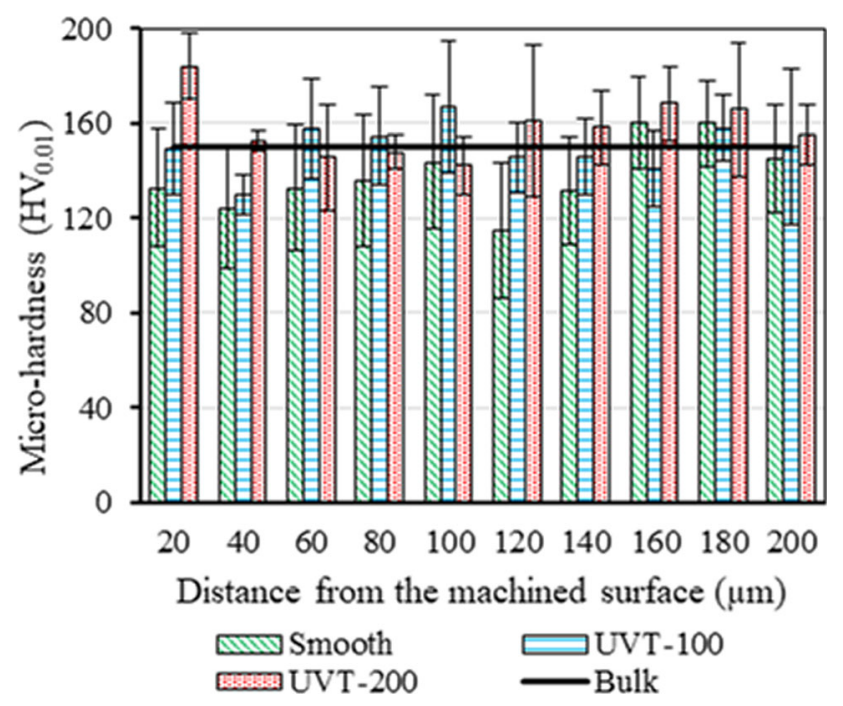

Fig. 4. Ti6Al4V micro-hardness as a function of the distance from the machined surface
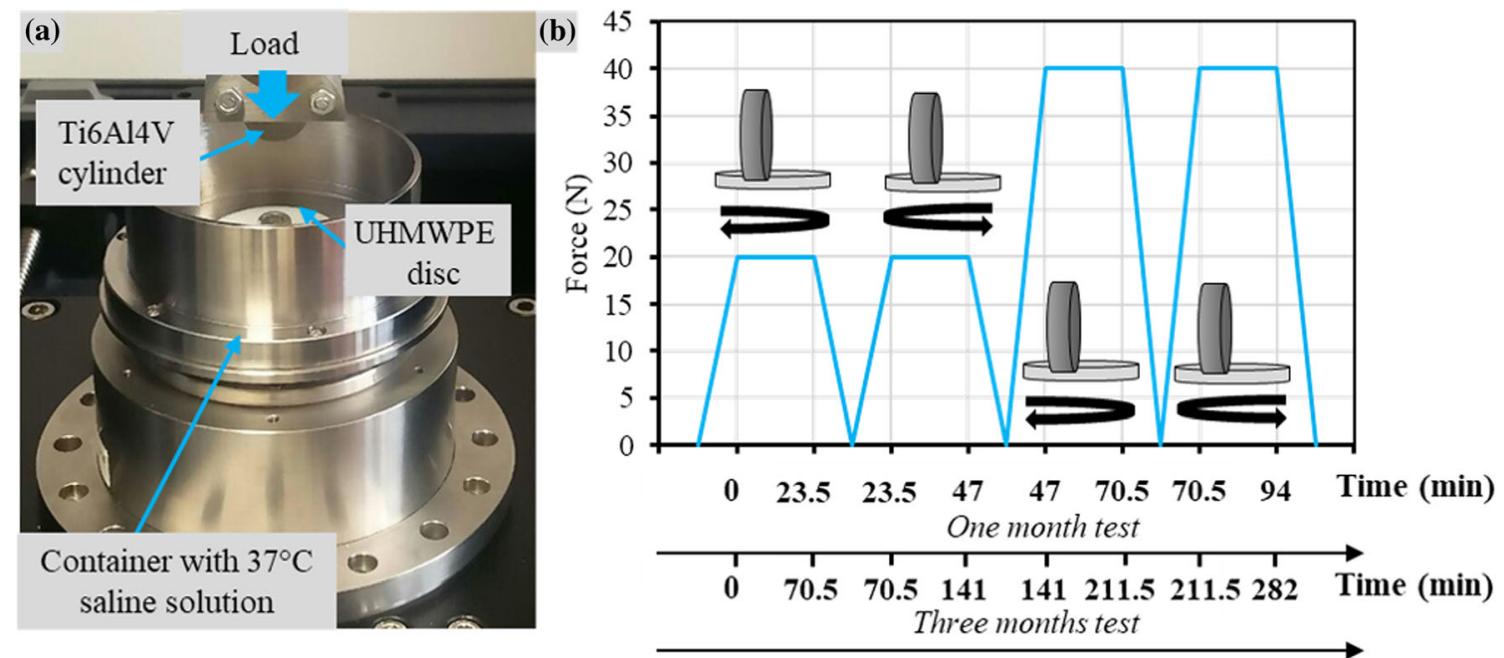

Fig. 3. (a) Experimental setup for wear testing; (b) scheme of the adopted wear tests 

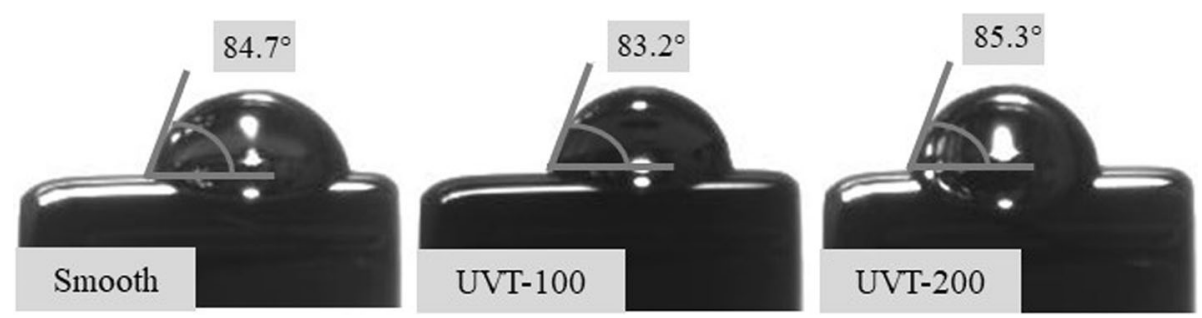

Fig. 5. Water droplets on the surfaces of the Ti6Al4V machined samples
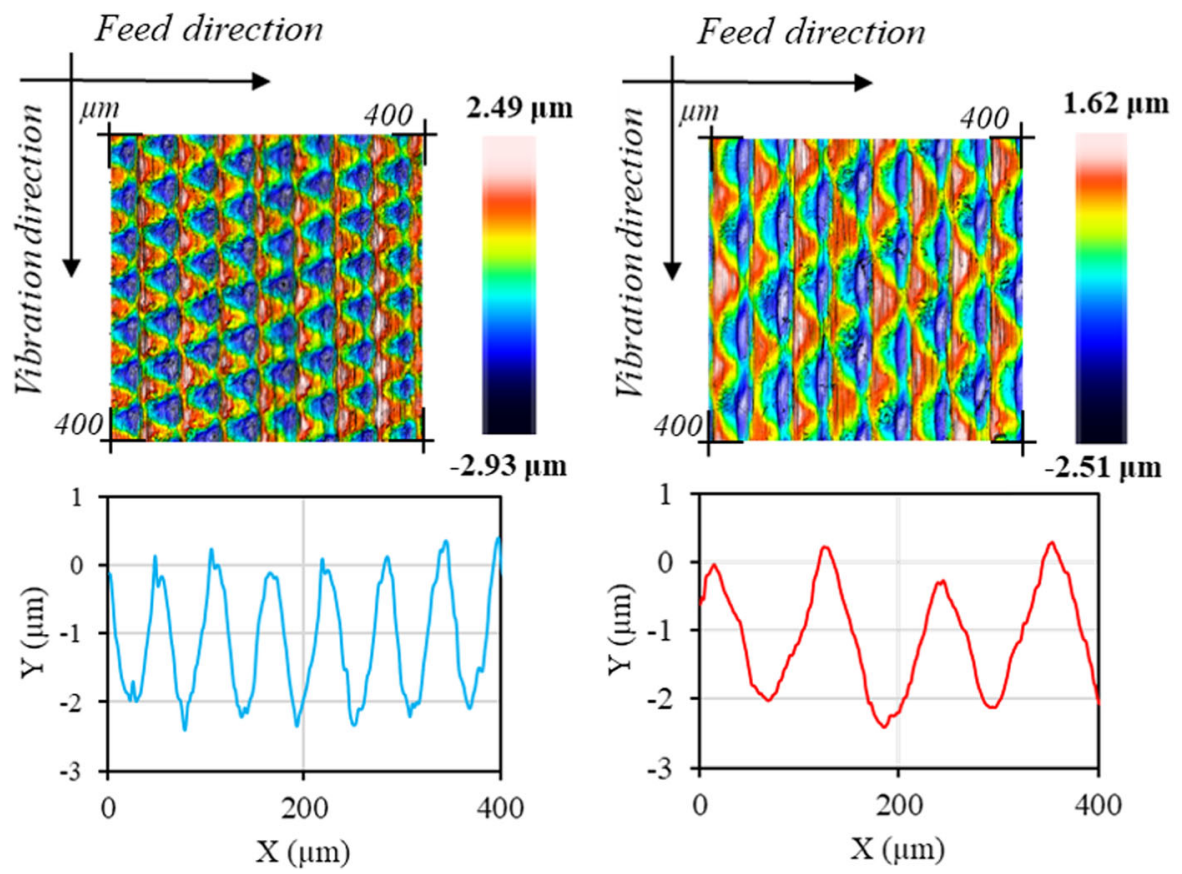

Fig. 6. 3D topographies and relative $2 \mathrm{D}$ profiles of the UVT-100 (left) and UVT-200 (right) samples before wear testing

Figure 6 shows partial profiles of the surfaces measured after machining to evidence the differences in the material distribution between the smooth, UVT-100, and UVT-200 samples. The cutting speed affects the distance between the dimples along the cutting direction, as predicted by $\mathrm{Eq} 1$. The distance between two subsequent dimples was $55 \mu \mathrm{m}$ for the UVT-100 sample and $111 \mu \mathrm{m}$ for the UVT-200 one. Figure 6 shows also how the surface texture was regular and the pattern repeated periodically, proving that the design requirements of the UVT system were respected.

Figure 7(a) reports the values of the surface texture parameters before wear testing. As expected, polishing induced a decrease in all the considered texture parameters, with the only exception of skewness, which set to a value comparable to that of the UVT-ed samples. It is worth noting that low skewness values are beneficial for wear applications (Ref 38). Focusing on the difference between the UVT-100 and UVT-200 samples, it can be seen that the former was characterized by both higher peaks (Spk) and deeper valleys (Svk) together with higher surface roughness Sa. The aspect ratio of the UVT-100 sample peaks was three times higher than the one obtained with a doubled cutting speed; this means that the peaks were leaner, confirming the surface texture data (see Table 3). The UVT-100 sample shows also a higher dimples density: this reflects well the fact that, in the same area, at a constant feed, the number of valleys was doubled compared to the one of the UVT-200 sample.

After wear testing, the smooth surface was subjected to a drastic increase in Sa (see Fig. $7 \mathrm{~b}$ and c), as a consequence of the presence of wear particles that tended to get trapped between the mating surfaces resulting in a third-body wear mechanism, thus provoking the surface roughness increase. On the contrary, both the UVT-100 and UVT-200 samples showed a reduction of $\mathrm{Sa}$ as a consequence of the flattening of the sliding interfaces (Ref 39].

For all the samples, regardless of the initial state condition, a drastic increase in the values of Spk and Svk was noticed after wear testing. By looking at the 2D profiles extracted from the surface topography of the UVT-100 and UVT-200 samples (Fig. 8), it can be seen that the wear development affected the profiles, which appeared patchier and more irregular than before wear testing. Again, the skewness of the smooth sample showed a peculiar behavior, settling to higher values compared to the one of the other samples. From these considerations, it can be concluded that, just after a month, the smooth surface changed completely its characteristics, achieving roughness values comparable to those of the UVT-100 and UVT-200 
$\square$ Smooth, before test

घUVT-100, before test

口UVT-200, before test

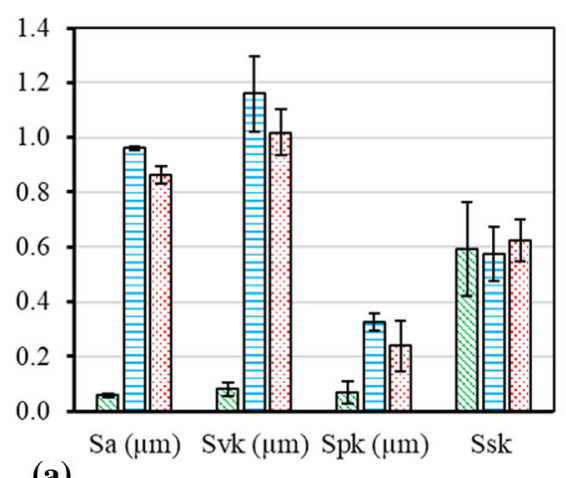

(a) $\square$ Smooth, after test (one month)

घUVT-100, after test (one month)

⿴囗十) UVT-200, after test (one month)

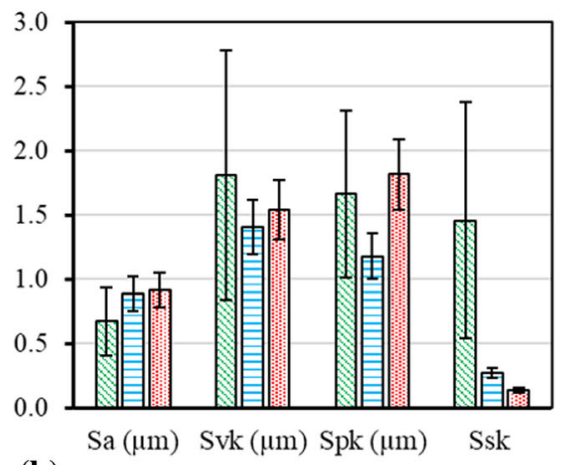

(b) $\square$ Smooth, after test (three months)

घUVT-100, after test (three months)

[0.6VT-200, after test (three months)

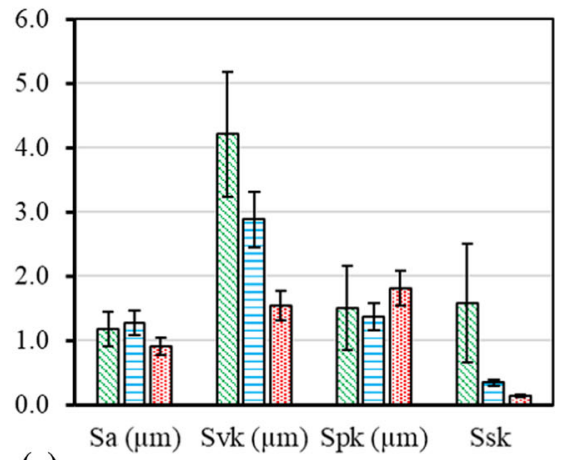

(c)

Testing time

Fig. 7. Surface texture parameters of the Ti6Al4V samples before and after wear testing

Table 3 Aspect ratio and density of the dimples of the UVT samples before and after wear testing

\begin{tabular}{lrrrrr}
\hline & \multicolumn{2}{c}{ Before wear testing } & & \multicolumn{2}{c}{ After one-month wear testing } \\
\cline { 2 - 4 } & \multicolumn{2}{c}{ AR } & Sdd & AR & \\
\hline UVT-100 & $0.036 \pm 0.005$ & $385 \pm 2$ & $0.041 \pm 0.001$ & \\
UVT-200 & $0.01 \pm 0.004$ & $189 \pm 9$ & $0.03 \pm 0.001$ & $\ldots$ \\
\hline
\end{tabular}

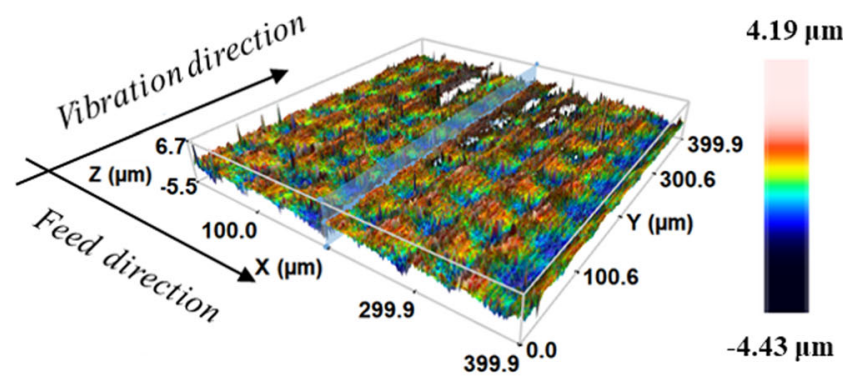

-UVT-100, before test

-UVT-100, after test (one month)



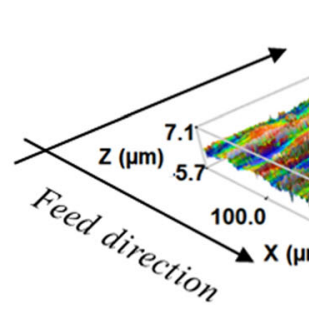

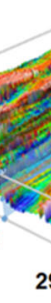

$\sin \left(\frac{\pi}{20}\right.$
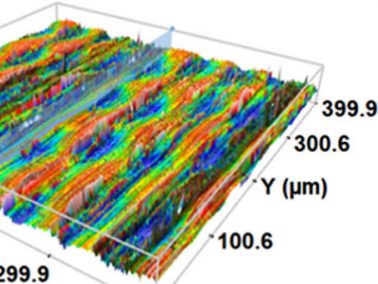

$399.9^{0.0}$

—UVT-200, before test

-UVT-200, after test (one month)

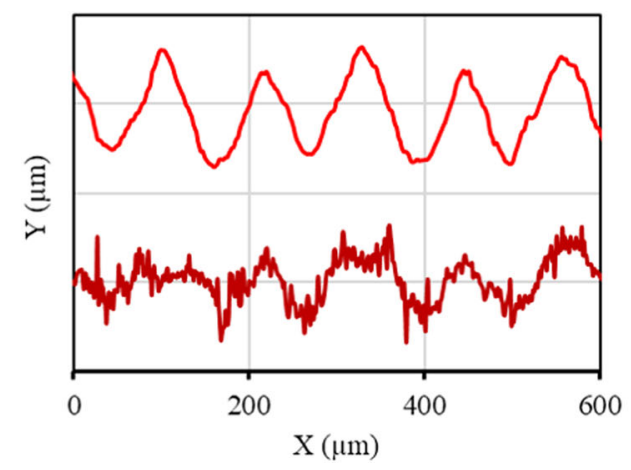

Fig. 8. 3D topographies and relative 2D profiles of the UVT-100 (left) and UVT-200 (right) samples after one-month wear testing 
samples. On the contrary, the unique surface texture generated by UVT was preserved.

After three-month wear testing, Sa and Ssk were very slightly influenced by the wear process compared to the ones after one-month wear testing: this was true for all the investigated samples. On the contrary, Spk and Svk were influenced by the wear process, being much more sensible to the abrasion and adhesion wear phenomena. In particular, a drastic increase in Spk was found for the UVT-200 sample, while a drastic increase in Svk was detected for both the smooth and UVT-100 samples.

Table 3 shows that the UVT-100 sample, characterized by higher AR values just after machining, preserved in a more efficient way its initial valleys configuration compared to the UVT-200 sample. As a consequence, it can preserve valleys for a longer time compared to the other sample. Actually, the AR increase after one-month wear testing is equal to $14 \%$ for the UVT-100 sample and $200 \%$ for the UVT-200 one. It is worth noting that the AR values after wear testing were affected by some uncertainty due to the samples jagged profiles, and, therefore, the reported values can be considered only indicative. This is the reason why AR and dimples density values were not calculated for the three-month wear tests.

The density of dimples Sdd showed a dramatic increase for both the textured samples after wear testing, which means that this parameter cannot be considered reliable for describing the characteristics of worn dimples. This can be explained with the fact that, by mirroring the surface to the $x$-axis, the presence of localized scratched areas played a major effect in the Sdd calculation. For this reason, the Sdd values after one moth wear testing are not reported in Table 3.

On the basis of the aforementioned results, UVT-100 condition appears to generate the surface texture that can be considered the most appropriate for the purpose of the study.

\subsection{Tribological Characteristics}

Table 4 shows the average Coefficient of Friction (COF) values acquired during wear testing at varying load and test duration. In case of the tests lasting one month with the application of a load of $20 \mathrm{~N}$, the UVT-100 and UVT-200 samples showed a COF reduction of $25 \%$ compared to the one of the smooth sample. The three-month wear tests showed a further reduction, which was more significant in case of the UVT-100 sample. When the load was increased to $40 \mathrm{~N}$, the UVT-100 and UVT-200 samples showed a COF reduction of 41 and $17 \%$ compared to the smooth one, respectively. The approximately same reduction in COF was found in the case of tests lasting three months and with the same applied load. In general, it was seen that the smooth surface always presented the highest friction coefficient regardless of the applied load.

Table 4 Average COF as a function of load and test duration

\begin{tabular}{lcrrrr}
\hline & \multicolumn{2}{c}{ One-month wear testing } & & \multicolumn{2}{c}{ Three-month wear testing } \\
\cline { 2 - 3 } \cline { 5 - 6 } & $\mathbf{2 0 ~ N}$ & $\mathbf{4 0 ~ N}$ & & $\mathbf{2 0 ~ N}$ & $\mathbf{4 0 ~ N}$ \\
\hline Smooth & $0.16 \pm 0.05$ & $0.17 \pm 0.01$ & & $0.21 \pm 0.02$ & $0.19 \pm 0.001$ \\
UVT-100 & $0.12 \pm 0.04$ & $0.1 \pm 0.02$ & & $0.13 \pm 0.01$ & $0.13 \pm 0.001$ \\
UVT-200 & $0.12 \pm 0.02$ & $0.14 \pm 0.05$ & & $0.1 \pm 0.08$ & $0.16 \pm 0.002$ \\
\hline
\end{tabular}

Lower friction coefficients are beneficial for biomedical bearings, since they reduce the frictional force responsible of aseptic loosening (Ref 40). It is well known that friction is influenced by both the surface texture and mechanical properties of the surface and sub-surface (Ref 41). As it was shown in $\S 3.1$, the micro-hardness of the UVT samples was comparable to that of the smooth sample; therefore, the COF decrease is found when testing UVT samples can be attributable only to presence of a textured surface. Similarly, different degrees of wettability can induce differences in the friction coefficient. It was shown in (Ref 42) that, under the hydrophilic regime, different wettability degrees contributed to shift down to lower values the Stribeck curve, due to the thicker layer of the formed fluid film. Again, it was shown in $\$ 3.2$ that the contact angles did not substantially differ, meaning that the COF reduction can be solely attributed to the presence of a textured surface.

The following main effects are expected to occur to improve the tribological performance of textured surfaces, according to (Ref 43-45): (1) a properly textured surface can have the ability to trap wear particles, limiting the presence of debris between the two mating surfaces, which can cause abrasive or adhesive wear, and are involved in three body wear mechanisms; (2) dimples can act as an efficient reservoir, storing wear particles inside, thus making the reduction in abrasive wear conceivable. In addition, it was demonstrated in (Ref 46) that a temperature reduction took place in the case of textured surfaces in comparison with polished surfaces, thanks to the efficient lubrication at the contact points. The latter can cause a softening in the PE disks, increasing the amount of abrasive wear.

Table 4 shows that the UVT samples are sensitive to the load applied during wear testing. Actually, when the applied load was $20 \mathrm{~N}$, the UVT samples showed comparable performances, while, when doubling the load, the COF of the UVT-100 sample was always lower than the one of the UVT-200 sample. This implies a different load-carrying capacity of the tested surfaces, which may be linked to the higher density of dimples of the UVT-100 sample (see Table 3). Chyr et al. (Ref 10) demonstrated that the presence of a microstructure on the surface could increase the load-carrying capacity of the lubricant film between two bearing surfaces in relative motion thanks to the increase in the lubricant film thickness and consequent reduction in contact, thereby reducing friction, and, potentially, wear of the articulating surfaces.

In addition to friction, wear occurring at the mating surfaces was evaluated by means of topography analyses. After the one-

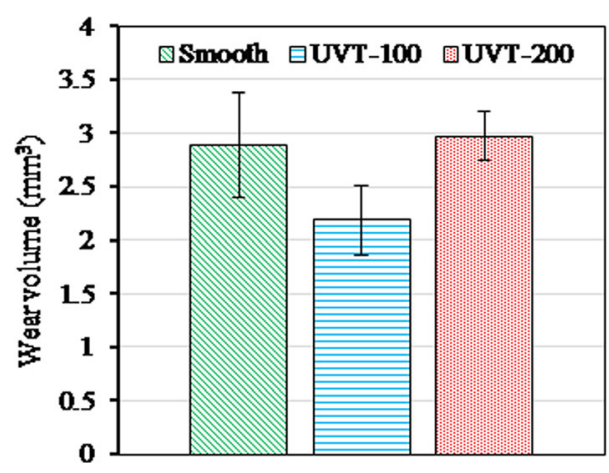

Fig. 9. Wear volume of the UHMWPE tested against smooth, UVT-100 and UVT-200 samples after three-month wear tests 

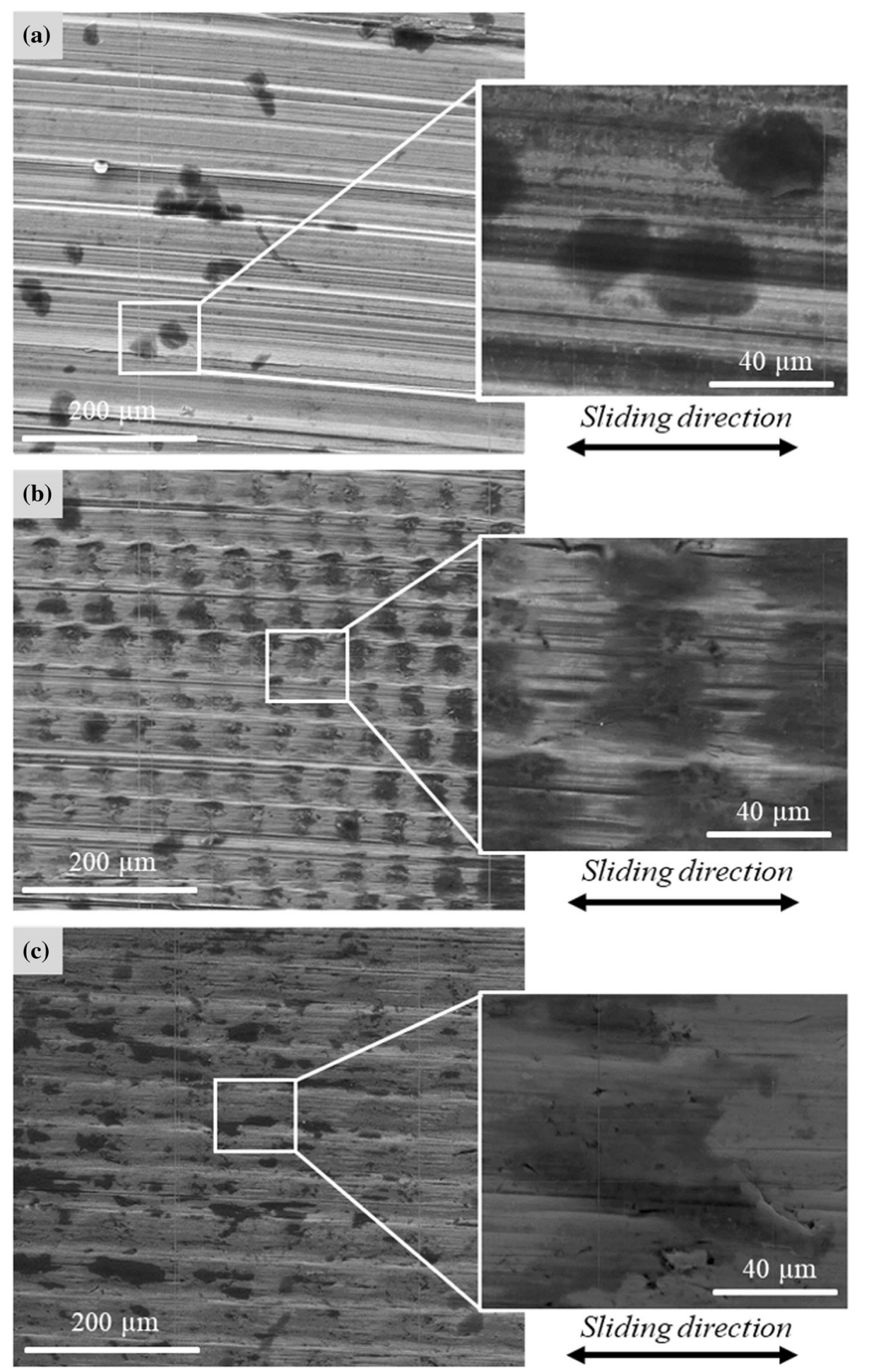

Fig. 10. SEM images at different magnification of the worn Ti6Al4V samples after three-month wear tests: (a) polished, (b) UVT-100, and c) UVT-200

month wear tests, only negligible levels of wear were recorded and, therefore, the wear volume calculation was not carried out. On the contrary, Fig. 9 reports the wear volume values of the UHMWPE after the three-month wear tests. Results show that the texturing of the Ti6Al4V surface did not alter the UHMWPE wear volume, since data referring to testing against smooth and UVT-200 samples set to comparable values. The wear volume of the UHMWPE tested against the UVT-100 sample, instead, was the lowest in accordance with the reported friction results.

In a wear process, only the summit points of the surface are in contact to each other, bearing the whole load. Over time and at increasing load, also the lower points of the surfaces come in contact due to the plastic deformation of the asperity areas. Although un-textured surfaces are smoother, the peaks that protrude above the mean line (described by the $S s k$ parameter) 
are demonstrated to increase as a consequence of the wear process. On the contrary, the Ssk values of the UVT samples decreased after wear testing, thanks to the presence of the dimples. In particular, the UVT-100 sample presented the highest initial $S d d$ that is demonstrated to be useful for decreasing friction and wear.

\subsection{Wear Scars}

Figure 10 reports SEM images at different magnification of the wear scars on the Ti6Al4V samples after three-months of wear testing. Abrasive wear signs appear as wear scars, clearly visible on the surface of the smooth sample (see Fig. 10a). As a consequence of the abrasion, flakes of material were removed from the surfaces, together with some wear debris. Actually, a large amount of wear debris particles was found on the smooth sample surface as evidenced in the magnified image of Fig. 10(a). These particles can favor the occurrence of the three-body abrasion phenomenon that may significantly increase the wear rate (Ref 39) and are detrimental for total hip replacements since they can act as foreign body stimulation, resulting in loosening of the implant. In addition, no sign of adhesive wear was noticeable.

On the contrary, wear debris can be trapped inside the valleys present on the UVT-ed surfaces. The magnification of Fig. 10(b) demonstrates that the surface valleys were completely full of wear particles preventing their harmful function. Traces of adhesive wear were also visible. The presence of adhesion wear can be considered positive as the adhered material can protect underlying layers from further abrasion,

Table 5 EDS point analysis of the light gray and dark gray zones of Fig.10

\begin{tabular}{lrrrrrr}
\hline & \multicolumn{7}{c}{ Element concentrations, wt\% } \\
\cline { 2 - 7 } & Ti & Al & V & C & O & Others \\
\hline Light gray & 91.5 & 5.1 & 3.4 & $\ldots$ & $\ldots$ & $\ldots$ \\
Dark gray & 7.4 & 2.4 & $\ldots$ & 71.8 & 18.2 & $<1$ \\
\hline
\end{tabular}
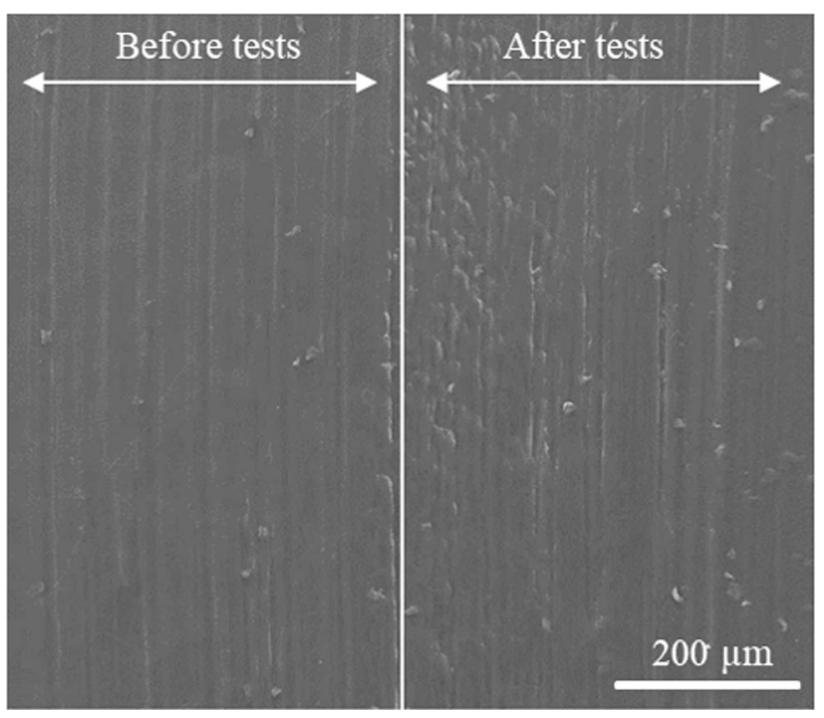

Fig. 11. SEM image of worn UHWPE after three-month wear tests (smooth Ti6Al4V as counterpart material) reducing the amount of the wear particles that can dissipate into the human body and increasing their size. EDS analyses were performed on both the light gray and dark gray regions visible in Fig. 10, and related results are shown in Table 5. The light gray zones were identified as material of the pin (Ti6Al4V), whereas the dark gray zones were identified as primarily coming from the material counterpart (UHMWPE).

The UVT-100 samples seem to have the most effective texture since the wear particles were collected in a more homogeneous way (see Fig. 10b compared to Fig. 10c). This can be attributed to the preservation of the starting texture for a longer duration as seen in the $2 \mathrm{D}$ profiles of Fig. 8 .

Figure 11 shows the comparison between the UHWPE before and after wear tests using the Ti6Al4V smooth surface as counterpart material. After testing, parallel ripples with plastically deformed edges together with a lot of wear particles were shown. In highly deformed areas, elongated fibrils extended from the surface (Ref 47).

No significant differences in the UHMWPE wear scars were found at varying the texture of the counterpart material.

\section{Conclusions}

In the present study, the effect of surface texturing on the wear performances of the Ti6Al4V-UHMPE pair was evaluated. Ultrasonic Vibration Turning (UVT) was exploited to create dimples on the surface of Ti6Al4V samples, varying the cutting speed in order to modify the distance between subsequent dimples.

Cylinder-on-disk wear tests were used with the objective of evaluating if the surface texture induced by UVT affected the tribological performances. Smooth surfaces produced by polishing were also tested for sake of comparison.

The main results can be summarized as follows:

- UVT did not affect the Ti6A14V characteristics in terms of micro-hardness and wettability.

- Even if the surface finish of the investigated samples after machining was very different, just after a month of wear testing, smooth samples showed a surface roughness comparable to the one of the UVT-ed samples indicating that the smooth texture was lost after few wear cycles.

- After a month of wear testing, 2D profiles of the worn surfaces of the UVT-ed samples showed that the dimples were partially preserved; therefore, their function was still active. Samples machined by UVT at the lowest cutting speed performed better, thanks to their higher initial aspect ratio and higher density of dimples.

- UVT-ed samples showed a significant reduction in the coefficient of friction compared to the smooth one, especially the sample machined at the lowest cutting speed.

- Wear volume of the UHMWPE was the lowest for the UVT-100 texture surfaces.

- SEM images of the wear scars after three months of wear testing confirmed that the dimples generated through UVT were filled of the counterpart material. This proves the dimples' efficiency in trapping the wear debris.

- UVT-ed samples were characterized by both abrasive and adhesive wear; the presence of the latter can be regarded as positive since it prevents the release of small wear debris particles in the human body. On the contrary, smooth 
surfaces were mostly characterized by abrasive wear and the presence of a higher amount of wear particles.

On these bases, it can be concluded that UVT can be a potential technology to machine surfaces for biomedical applications.

\section{Funding}

Open access funding provided by Università degli Studi di Padova within the CRUI-CARE Agreement.

\section{Open Access}

This article is licensed under a Creative Commons Attribution 4.0 International License, which permits use, sharing, adaptation, distribution and reproduction in any medium or format, as long as you give appropriate credit to the original author(s) and the source, provide a link to the Creative Commons licence, and indicate if changes were made. The images or other third party material in this article are included in the article's Creative Commons licence, unless indicated otherwise in a credit line to the material. If material is not included in the article's Creative Commons licence and your intended use is not permitted by statutory regulation or exceeds the permitted use, you will need to obtain permission directly from the copyright holder. To view a copy of this licence, visit http://creativecommons.org/licenses/by/4.0/.

\section{References}

1. A. Molinari, G. Straffellini, B. Tesi and T. Bacci, Dry Sliding Wear Mechanisms of the Ti6Al4V Alloy, Wear, 2010, 208(1-2), p 105-112

2. G. Straffelini and A. Molinari, Dry Sliding Wear of Ti-6Al-4V Alloy as Influenced by the Counterface and Sliding Conditions, Wear, 1999, 236(1/2), p 328-338

3. H. Guleryuz and H. Cimenoglu, Effect of Thermal Oxidation on Corrosion and Corrosion-Wear Behaviour of a Ti-6Al-4V Alloy, Biomaterials, 2004, 25, p 3325-3333

4. P. Majumdar, S.B. Singh and M. Chakraborty, Wear Response of HeatTreated Ti-13Zr-13Nb Alloy in Dry Condition and Simulated Body Fluid, Wear, 2008, 264, p 1015-1025

5. A.P. Serroa, C. Completo, R. Colaço, F. Dos Santos, C. Lobato da Silva, J.M.S. Cabral, H. Araújoe, E. Pires, B. Saramago, A Comparative Study of Titanium Nitrides, TiN, TiNbN and TiCN, as Coatings for Biomedical Applications, Surf. Coat. Technol., 2009, 03, 37013707

6. M. Kheradmandfar, S.F.K. Bozorg, J.S. Lee, Z.H. Hanzaki, Y.S. Pyund, S.W. Cho, A. Amanov and D.E. Kim, Significant Improvement in cell Adhesion and Wear Resistance of Biomedical-Type Titanium Alloy Through Ultrasonic Nanocrystal Surface Modification, J. Alloys Compd., 2018, 762, p 941-949

7. S. Ghosh and S. Abanteriba, Status of Surface Modification Techniques for Artificial Hip Implants, Sci. Technol. Adv. Mater. Sci., 2016, 17(1), p 715-735

8. M. Ito, M. Kaneda, T. Yutha, I. Nishimura, K. Yasuda, M.D. Matsuno, Reduction of Polyethylene Wear by Concave Dimples on the Frictional Surface in Artificial Hip Joints, J. Arthoplast., 2000, 15, 332-338

9. H. Sawano, S. Warisisawa and S. Ishihara, Study on Long Life of Artificial Joints by Investigating Optimal Sliding Surface Geometry for Improvement in Wear Resistance, Precis. Eng., 2009, 33, p 492-498

10. A. Chyr, M. Qiu, J.W. Speltz, R.L. Jacobsen, A.P. Sanders and B. Raeymaekers, A patterned Microtexture to Reduce Friction and Increase Longevity of Prosthetic Hip Joints, Wear, 2014, 315, p 51-57

11. T.C. Hu, L.T. Hu, Q. Ding, The Effect of Laser Surface Texturing on the Tribological Behavior of Ti-6Al-4V, Proc. Inst. Mech. Eng. Part J J. Eng. Tribol., 2012, 226, 854-863
12. A.Y. Fasasi, S. Mwenifumbo, N. Rahbar, J. Chen, M. Li, A.C. Beye, W.O. Soboyejo, Nano-Second UV Laser Processed Micro-Grooves on Ti6Al4V for Biomedical Applications, Mater. Sci. Eng. C, 2009, 29(1), 5-13

13. D. Choudhury, H.A. Ching, A.B. Mamat, J. Cizek, N.A. Abu Osman, M. Vrbka, M. Hartl, I. Krupka, Fabrication and Characterization of DLC Coated Microdimples on Hip Prosthesis Heads, J. Biomed. Mater. Res. B, 2015, 103(5), 1002-1012

14. A. Kurella and N.B. Dahotre, Review Paper: Surface Modification for Bioimplants: the Role of Laser Surface Engineering, J. Biomater. Appl., 2005, 2(1), p 5-50

15. R.M. Kumar, P. Gupta, S. Kumar, A. Mittal and M. Shekhar, Sustained Drug Release from Surface Modified UHMWPE for Acetabular Cup Lining in Total Hip Implant, Mater. Sci. Eng. C., 2017, 77, p 649-661

16. Y. Zhao, B.R. Mcleod, N. Balaban, F.Z. Hu, N.G. Sotereanos, J.W. Costerton, P.S. Stewart, J.C. Post and Q. Lin, Engineering Approaches for the Detection and Control of Orthopaedic Biofilm Infections, Clin. Orthop. Relat. Res., 2005, 437, p 59-66

17. A. Greco, S. Raphaelson, K. Ehmann, Q.J. Wang, C. Lin, Surface Texturing of Tribological Interfaces Using the Vibromechanical Texturing Method, J. Manuf. Sci. Eng., 2009, 131(6), 061005

18. X. Liu, D. Wu, J. Zhang, X. Hu and P. Cui, Analysis of Surface Texturing in Radial Ultrasonic Vibration-Assisted Turning, J. Mater. Process Technol., 2017, 267, p 186-195

19. P. Guo and K.F. Ehmann, An Analysis of the Surface Generation Mechanics of the Elliptical Vibration Texturing Process, Int. J. Mach. Tools Manuf., 2013, 64, p 85-95

20. K.N. Prasad, I.. Syed, S.K. Subbu, Laser Dimple Texturing-Applications, Process, Challenges, and Recent Developments: a Review, Aust. J. Mech. Eng., 2019, 1-16

21. Q. Allen, B. Raeymaekers, Surface Texturing of Prosthetic Hip Implant Bearing Surfaces: a Review, J. Tribol., 2021, 143(4), 040801

22. S. Amini, H.N. Hosseinabadi and A. Sajjady, Applied Surface Science Experimental Study on Effect of Micro Textured Surfaces Generated by Ultrasonic Vibration Assisted Face Turning on Friction and Wear Performance, Appl. Surf. Sci., 2016, 390, p 633-648

23. D. Xing, J. Zhang, X. Shen, Y. Zhao and T. Wang, Tribological Properties of Ultrasonic Vibration Assisted Milling Aluminium Alloy Surfaces, Procedia CIRP, 2013, 6, p 539-544

24. E. Di. Iorio, R. Bertolini, S. Bruschi and A. Ghiotti, Design and Development of an Ultrasonic Vibration Assisted Turning System for Machining Bioabsorbable Magnesium Alloys, Procedia CIRP, 2018, 77, p 324-327

25. K.L. Menzies and L. Jones, The Impact of Contact Angle on the Biocompatibility of Biomaterials, Optom. Vis. Sci., 2010, 87(6), p 387399

26. R. Shi, B. Wang, Z. Yan, Z. Wang and L. Dong, Effect of Surface Topography Parameters on Friction and Wear of Random Rough Surface, Materials, 2019, 12(17), p 2762

27. ISO 25178-1:2016, Surface Texture: Areal Part 1: Indication of Surface Texture, 2016

28. S. Bruschi, R. Bertolini, F. Medeossi, A. Ghiotti, E. Savio, Case Study: The Application of Machining-Conditioning to Improve the Wear Resistance of Ti6A14V Surfaces for Human Hip Implants, Wear 2018, 394-395, 134-142

29. B.S. Ramamurti, C.R. Bragdon, D.O. O'Connor, J.D. Lowenstein, M. Jasty, D.M. Estok and W.H. Harris, Loci of Movement of Selected Points on the Femoral Head During Normal Gait, J. Arthroplast., 1996, 11, p 845-852

30. A. Wang, A Unified Theory of Wear for Ultra-High Molecular Weight Polyethylene in Multi-Directional Sliding, Wear, 2001, 248, p 38-47

31. E.Y. Gutmanas and I. Gotman, PIRAC Ti Nitride Coated Ti-6Al-4V Head Against UHMWPE Acetabular Cup-Hip Wear Simulator Study, J. Mater. Sci. Mater. Med., 2004, 15(4), p 327-330

32. J. Kenneth Langstreth, Contact Mechanics, Cambridge university press, 1987

33. A. Borruto, A New Material for Hip Prosthesis Without Considerable Debris Release, Med. Eng. Phys., 2010, 32, p 908-913

34. ISO 7206-3:1988 Orthopaedic Joint Prostheses-Method for Determination of Endurance Properties of Stemmed Femoral Components of Hip Joint Prostheses Without Application of Torsion, 1990

35. D.T. Tsukayama, R. Estrada and R.B. Gustilo, A Study of the Treatment of One Hundred and Six Infections, J. Bone Joint Surg. Am., 1996, 78, p 512-523 
36. R.A. Gittens, L. Scheideler, F. Rupp, S.L. Hyzy, J. Geis-Gerstorfer, Z Schwartz and B.D. Boyan, A Review on the Wettability of Dental Implant Surfaces II: Biological and Clinical Aspects, Acta Biomater. 2014, 10, p 2907-2918

37. Y. Lu, P. Guo, P. Pei and K.F. Ehmann, Experimental Studies of Wettability Control on Cylindrical Surfaces by Elliptical Vibration Texturing, Int. J. Adv. Manuf. Technol., 2014, 76, p 1807-1817

38. R. Leach, Characterisation of Areal Surface Texture, Springer, 2013, 1

39. S. Bruschi, R. Bertolini, A. Bordin, F. Medea and A. Ghiotti, Influence of the Machining Parameters and Cooling Strategies on the Wear Behavior of Wrought and Additive Manufactured Ti6A14V for Biomedical Applications, Tribol. Int., 2016, 102, p 133-142

40. J.L. Prudhon, R. Verdier and H.J. Caton, Low friction Arthroplasty and Dual Mobility Cup: a New Gold Standard, Int. Orthop., 2017, 41, p 563-571

41. J.F. Archard, Contact and Rubbing of Flat Surfaces, J. Appl. Phys., 1953, 24, p 981

42. W. Huang, L. Jiang, C. Zhou and X. Wang, The Lubricant Retaining Effect of Micro-dimples on the Sliding Surface of PDMS, Tribol. Int., 2012, 52, p 87-93
43. A.A.G. Bruzzone, H.L. Costa, P.M. Lonardo and D.A. Lucca, Advances in Engineered Surfaces for Functional Performance, CIRP Ann-Manuf. Technol., 2008, 57, p 750-769

44. D.E. Kim, K.M. Cha, I. Sung and J. Bryan, Design of Surface Microstructures for Friction Control in Micro-systems Applications, CIRP Ann. Manuf. Technol., 2002, 51, p 495-498

45. A.A.G. Bruzzone and H.L. Costa, Functional Characterization of Structured Surfaces for Tribological Applications, Procedia CIRP., 2013, 12, p 456-461

46. U. Sudeep, R.K. Pandey and N. Tandon, Effects of Surface Texturing on Friction and Vibration Behaviors of Sliding Lubricated Concentrated Point Contacts Under Linear Reciprocating Motion, Tribol. Int., 2013, 62, p 198-207

47. A. Elfick, Influence of Crosslinked Polyethylene Structure on Wear of Joint Replacements, Tribol. Int., 2009, 42, p 1582-1594

Publisher's Note Springer Nature remains neutral with regard to jurisdictional claims in published maps and institutional affiliations. 